\title{
Methanol, Paracetamol Toxicities and Acute Blindness
}

\author{
Aldanah Althwanay ${ }^{1}$, Manar M. Alharthi ${ }^{2}$, Mohammad Aljumaan ${ }^{3}$, Yousef Almubarak ${ }^{1}$, Abdullah Alamri \\ 4
}

1. Internal Medicine, Imam Abdulrahman Bin Faisal University, Khobar, SAU 2. Internal Medicine, King Fahad Specialist Hospital, Dammam, SAU 3. Emergency Medicine, Imam Abdulrahman Bin Faisal University, Khobar, SAU 4. Neurology, Imam Abdulrahman Bin Faisal University, Khobar, SAU

Corresponding author: Abdullah Alamri, neurologist.neurointensivist@gmail.com

\begin{abstract}
Methanol toxicity remains a common problem in developing countries including Saudi Arabia. However, it is much less available than other toxins; thus, clinical suspicion and correlation play pivotal roles in diagnosis. On the other hand, paracetamol is widely available and overdose/toxicity is highly suspected especially in young females. Diagnosis of methanol toxicity can be difficult in cases where history is not readily available and it requires a high degree of suspicion, especially when ingestion of another substance is revealed first as should not preclude the possibility of co-ingestion.
\end{abstract}

We report a case of a medically free 26 year old woman who was brought to the Emergency Department (ED) of our hospital with a history of ingestion of a massive amount of paracetamol tablets and a concurrent abdominal pain with vomiting. After arrival, she became unresponsive with Glasgow Coma score (GCS) of $8 / 15$ and was mechanically ventilated. Initial laboratory investigations identify the paracetamol level of 1200 $\mathrm{ug} / \mathrm{ml}$ and a significantly high anion gap metabolic acidosis. Owing to the suspicious circumstances and the depth of acidosis, a co-ingestion of methanol and ethylene glycol was suspected. Upon further evaluation, osmolal gap was found to be significantly high as well. Since neither volatile compound screening nor Fomepizole were available in our hospital, an urgent $\mathrm{N}$-acetylcysteine and sodium bicarbonate $\left(\mathrm{NaHCO}_{3}\right)$ were started alongside hemodialysis. Subsequent brain computed tomography (CT) and magnetic reasoning imaging (MRI) revealed changes in putamen and basal ganglia most consistent with methanol toxicity. She was successively extubated on day four of hospitalization with residual visual deficits that had resolved eventually after several follow-ups.

Categories: Internal Medicine, Neurology

Keywords: methanol toxicity, paracetamol toxicity, blindness, coma, dialysis

\section{Introduction}

Received 02/17/2020

Review began 03/26/2020 Review ended 05/12/2020 Published 05/18/2020

\section{() Copyright 2020}

Althwanay et al. This is an open access article distributed under the terms of the Creative Commons Attribution License CC-BY 4.0., which permits unrestricted use, distribution, and reproduction in any medium, provided the original author and source are credited.
Methanol is a clear and colorless form of toxic alcohol. In the developing countries including Saudi Arabia, it can be found as a solvent in some brands of perfume and cologne, handmade liquor and smuggled alcohol. Ingestion of methanol may be accidental or as a suicidal attempt [1]. Methanol toxicity remains a common problem, however, it is much less available than other toxins; thus, clinical suspicion and correlation play pivotal roles in diagnosis. On the other hand, paracetamol is widely available and overdose/toxicity is highly suspected especially in young females. The presentation of methanol toxicity can be vary from mild to lifethreatening symptoms similar to that of paracetamol toxicity; hence symptoms may overlap and lead to a premature diagnosis of ingestion of a single specific substance without ruling out the possibility of coingestion of a single specific substance and the consequences of their interactions. The literature does not discuss the possible consequences and interactions of methanol and paracetamol co-ingestion. However, an article published in 2008 revealed that the co-ingestion of paracetamol and ethanol, which is another form of alcohol, actually limits the effect of toxicity of both substances together [2]. Exposure to methanol can cause a wide range of neurological manifestations, most commonly confusion, coma and vision loss and less frequently tremor as a manifestation of putaminal hemorrhage/necrosis. Reports have indicated that death from methanol toxicity had ranged from $8-36 \%$ and permanent loss of vision has been observed in $20-40 \%$ of patients who survive the acute injury [3-5]. Paracetamol overdose can cause non-specific symptoms within the first 24 hours, including fatigue, abdominal pain, or nausea. After several days, patients may complain of jaundice, blood clotting problems, and confusion that occurs as a result of liver failure. Additional complications may include kidney failure, pancreatitis, low blood sugar, and lactic acidosis [6].

\section{Case Presentation}

A 26 year old healthy female presented to our ED with a one-day history of a massive amount of paracetamol tablets ingestion and a concurrent abdominal pain with vomiting. Her condition promptly deteriorated and she became confused and unresponsive. Upon presentation, her blood pressure was 93/60 $\mathrm{mmHg}$, pulse rate was $130 \mathrm{bpm}$, respiratory rate was $30 \mathrm{Bpm}$, SaO2 was $87 \%$ on room-air, random blood sugar was $140 \mathrm{mg} / \mathrm{dl}$ and GCS of 8/15. On examination, she had fixed bilaterally dilated pupils (6 mm). A 


\section{Cureus}

baseline electrocardiogram (ECG) was taken and showed supraventricular tachycardia alongside hemodynamic instability for which she received two synchronized cardioversion shocks. She was immediately resuscitated with intravenous fluid and was intubated. Initial laboratory results including liver function test were within acceptable ranges except for $\mathrm{pH}(6.68)$, anion gap $(31 \mathrm{mmol} / \mathrm{L})$, delta anion gap $(0.9$ $\mathrm{mmol} / \mathrm{L}), \mathrm{PCO} 2(24.7 \mathrm{mmHg}), \mathrm{HCO}_{3}:(3.9 \mathrm{mmol} / \mathrm{L})$, creatinine level $(1.5 \mathrm{mg} / \mathrm{dl})$, potassium $(6.6 \mathrm{mmol} / \mathrm{L})$, lactic acid $(6.4 \mathrm{mmol} / \mathrm{L})$, urine ketones $(15 \mathrm{mg} / \mathrm{dl})$. Urine toxicology for was positive for acétaminophèn (1200 $\mathrm{ug} / \mathrm{ml}$ after 12 hours of ingestion) and salicylate (less than $0.2 \mathrm{ug} / \mathrm{ml}$ ), but was negative for opiates. The presence of high anion gap metabolic acidosis was initially thought to be secondary to ketoacidosis and hyperlactatemia. However, the degree of lactic acidosis and ketonuria was not high enough to explain the extent of the acidosis and on further evaluation, osmolal gap was calculated to be $34.1 \mathrm{mmol} / \mathrm{L}$. Since the osmolal gap was high, toxic alcohol ingestion (most probably methanol) was the working diagnosis owing to the dubious circumstances and clinical presentation. Methanol level was not obtained as our hospital lacks a volatile compound screening test. A head CT followed by MRI were obtained after initiating treatment and revealed putaminal and basal ganglia necrosis consistent with methanol toxicity (Figure 1,2).

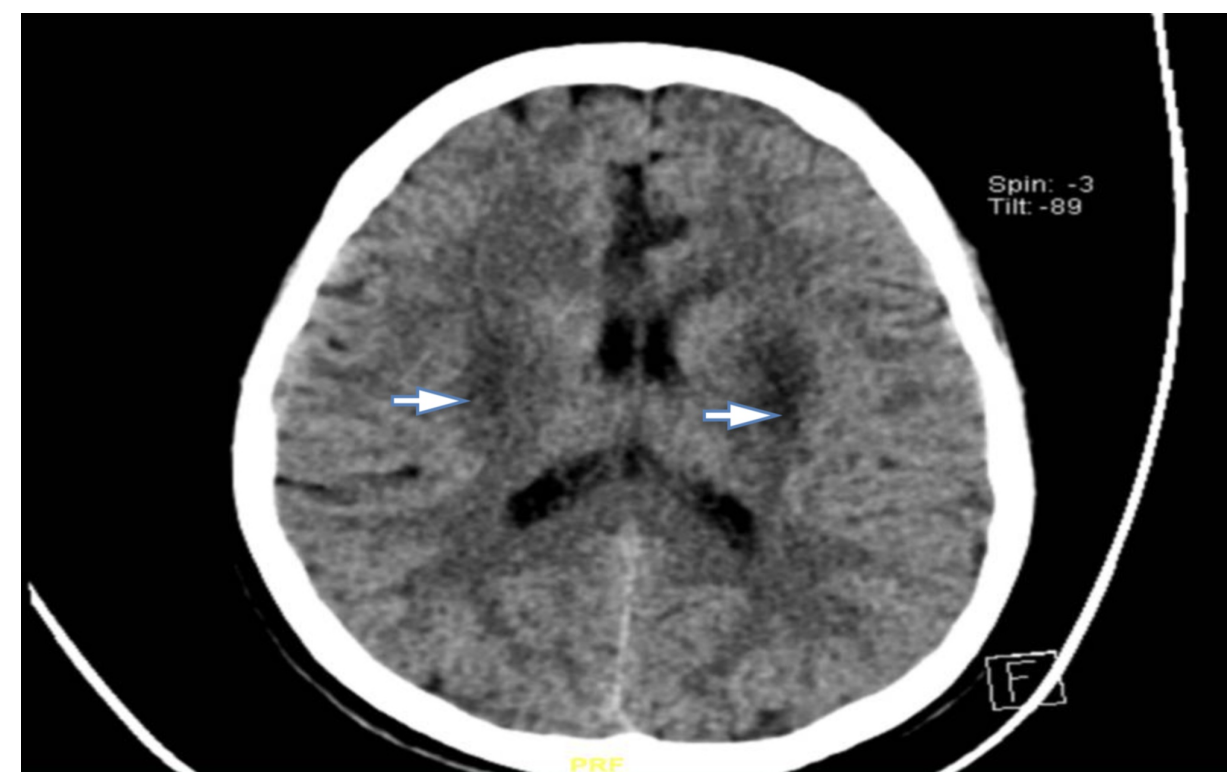

FIGURE 1: Non-enhanced CT scan of the brain showing bilateral basal ganglia hypodensities

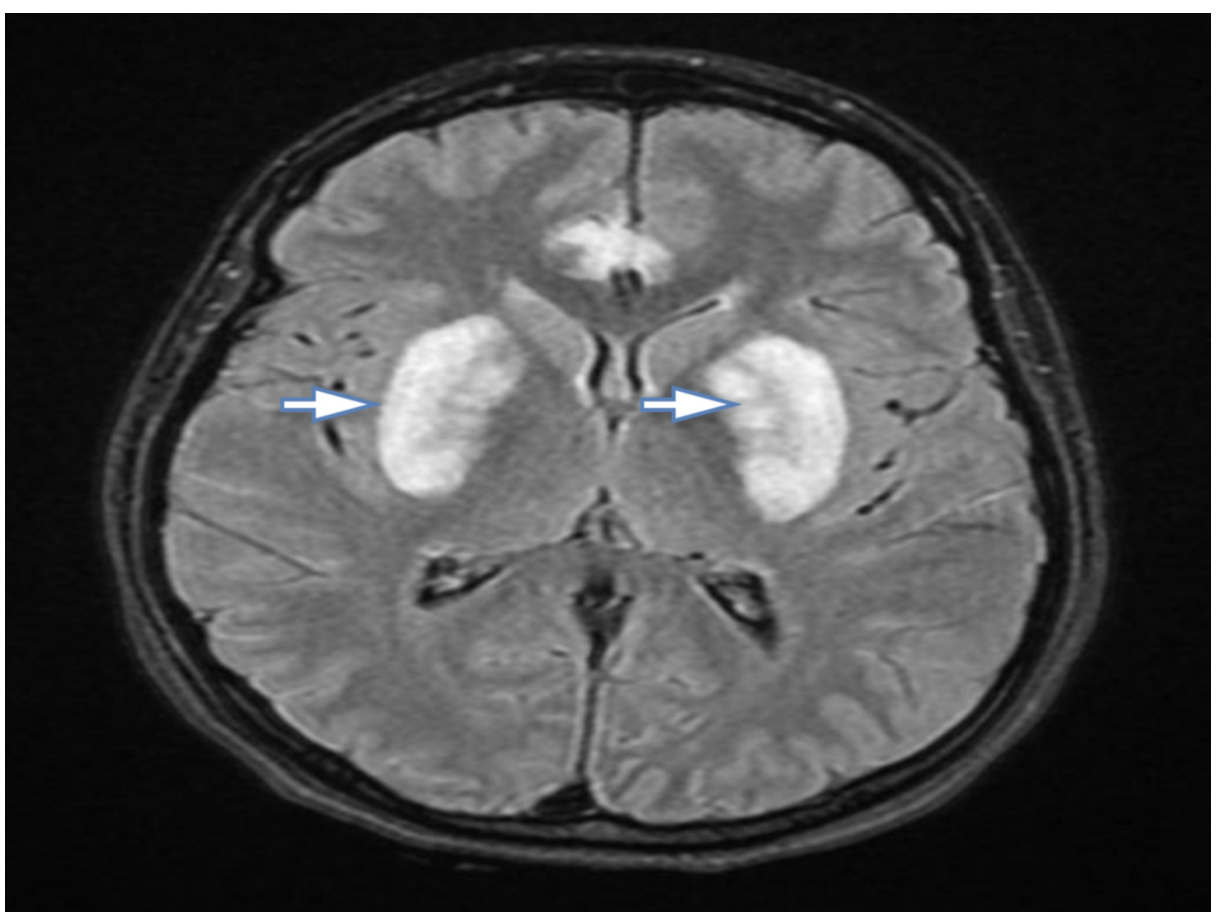


As the patient had severe metabolic acidosis with $\mathrm{pH}<7.1$, she was given NaHCO3 infused with $5 \%$ dextrose and was put on urgent hemodialysis to clear the methanol as Fomepizole is not available in our hospital regularly. In addition to that, she received $\mathrm{N}$ - acetylcysteine for paracetamol toxicity and was admitted to the Intensive Care Unit (ICU). On the second day of her admission and during the time of sedation vacation, visual threat examination revealed vision loss and the ophthalmologist confirmed the diagnosis of toxic optic neuropathy. On the fourth day, successful extubation was achieved with residual visual deficits. Her blood $\mathrm{pH}$, anion gap and osmolal gap levels had normalized eventually. Follow up appointments with neurology, psychiatry and ophthalmology clinics were recommended. After several follow-ups, she regained her full vision.

\section{Discussion}

Methanol toxicity remains a common problem in developing countries including Saudi Arabia. Methanol gets oxidized by alcohol dehydrogenase to formaldehyde, which is oxidized further to formic acid by formaldehyde dehydrogenase. Formic acid accumulates are responsible for metabolic acidosis in methanol toxicity cases and there is a direct correlation between formic acid concentration and incidence of morbidity and mortality. Formic acid inhibits cytochrome oxidase and is the cause of ocular toxicity [7]. The presentation of methanol toxicity can vary from mild symptoms to life-threatening. At first, symptoms of methanol toxicity could be similar to alcohol toxicity. For instance, the patient may present with ataxia, disinhibition, nausea, vomiting, and epigastric abdominal pain. Drowsiness could rapidly progress to seizure and coma in later stages due to metabolic acidosis indirectly or by direct damage to the brain parenchyma, eventually requiring intubation [8]. Likewise, methanol toxicity may present similar to paracetamol toxicity, where patients present with abdominal pain, nausea, vomiting and in severe cases metabolic acidosis secondary to lactic acidosis [6]. Our patient, for instance, presented with abdominal pain and vomiting alongside hemodynamic instability. Her condition rapidly deteriorated where she became unresponsive and developed supraventricular tachycardia for which she was rapidly resuscitated with two synchronized cardioversion shocks in addition to intravenous fluid and endotracheal intubation.

One of the most common manifestations of methanol toxicity is ocular findings which include optic disc hyperemia, compromised pupillary response with little or no retinal damage [8]. Our patient had early presentation of fixed bilateral dilation of pupils. An ophthalmology examination had revealed bilateral vision loss. Serum methanol levels and arterial blood gas are crucial for diagnosis. Moreover, the anion gap and osmolal gap calculations are essential to differentiate between methanol toxicity and other causes of high anion gap metabolic acidosis such as ketoacidosis and lactic acidosis [9,10].

Both the high anion gap and osmolal gap suggested toxic alcohol ingestion such as methanol and ethylene glycol [11]. Diagnosis can be difficult in cases where history is not readily available and requires a high degree of suspicion, especially when ingestion of another substance is revealed first as it should not preclude the possibility of co-ingestion. Our patient had presented with paracetamol ingestion and coingestion of methanol was highly suspected, especially in such patients with high-risk behavior alongside the significantly high anion gap and osmolal gap which could be explained by the extent of neither ketoacidosis nor lactic acidosis.

Since none of the published literature discussed or reported the co-ingestion of paracetamol and methanol together, it is unknown if there was a possible interaction between them and whether it would affect the extent of toxicity of both substances negatively or positively.

Brain imaging may help by showing the characteristic findings of bilateral putaminal necrosis/ hemorrhage [8]. Nevertheless, these findings are not specific and can be seen with other diseases, such as Wilson's disease and stroke [12]. Prompt treatment with Fomepezole or hemodialysis is crucial as the degree and irreversibility of damage caused by formic acid is time-sensitive and the increased acidity facilitates the diffusion of formic acid into cells [8]. Thus, our patient received $\mathrm{NaHCO}_{3}$ to decrease the acidity as pH cutoff was $\leqslant 7.1$ and underwent hemodialysis to eliminate both methanol and co-ingested paracetamol alongside $\mathrm{N}$-acetylcysteine.

\section{Conclusions}

Diagnosis of methanol toxicity can be difficult in cases where history is not readily available and requires a high degree of suspicion, especially when another substance ingestion is revealed first as it should not preclude the possibility of co-ingestion. Prompt treatment with Fomepezole or hemodialysis is crucial as the degree and irreversibility of damage caused by formic acid is time-sensitive. 


\section{Additional Information}

\section{Disclosures}

Human subjects: Consent was obtained by all participants in this study. MORRE issued approval NA. Conflicts of interest: In compliance with the ICMJE uniform disclosure form, all authors declare the following: Payment/services info: All authors have declared that no financial support was received from any organization for the submitted work. Financial relationships: All authors have declared that they have no financial relationships at present or within the previous three years with any organizations that might have an interest in the submitted work. Other relationships: All authors have declared that there are no other relationships or activities that could appear to have influenced the submitted work.

\section{References}

1. Chan APL, Chan TYK: Methanol as an unlisted ingredient in supposedly alcohol-based hand rub can pose serious health risk. Int J Environ Res Public Health. 2018, 15:1440.

2. Waring W, Stephen A, Malkowska A, Robinson O: Acute ethanol coingestion confers a lower risk of hepatotoxicity after deliberate acetaminophen overdose. Acad Emerg Med. 2008, 15:54-58. 10.1111/j.15532712.2007.00019.x

3. Kraut JA, Mullins ME: Toxic alcohols. N Engl J Med. 2018, 18:270-280. 10.1056/NEJMra1615295

4. Ahmed F, Khan NU, Ali N, Feroze A: Methanol poisoning: 27 years experience at a tertiary care hospital . J Pak Med Assoc. 2017, 67:1751-1752.

5. Galvez-Ruiz A, Elkhamary SM, Asghar N, Bosley TM: Visual and neurologic sequelae of methanol poisoning in Saudi Arabia. Saudi Med J. 2015, 36:568-574.

6. Yoon E, Babar A, Choudhary M, Kutner M, Pyrsopoulos N: Acetaminophen-induced hepatotoxicity: a comprehensive update. J Clin Transl Hepatol. 2016, 4:131-142. 10.14218/JCTH.2015.00052

7. Barceloux DG, Bond GR, Krenzelok EP, Cooper H, Vale JA, American Academy of Clinical Toxicology Ad Hoc Committee on the Treatment Guidelines for Methanol Poisoning.: American Academy of Clinical Toxicology practice guidelines on the treatment of methanol poisoning. J Toxicol Clin Toxicol. 2002, 40:415-46. $10.1081 /$ clt-120006745

8. Methanol toxicity clinical presentation. (2018). Accessed: May 15, 2020: https://emedicine.medscape.com/article/1174890-clinical.

9. Jacobsen D, McMartin KE: Methanol and ethylene glycol poisonings. Mechanism of toxicity, clinical course, diagnosis and treatment. Med Toxicol. 1986, 1:309-34. 10.1007/BF03259846

10. Fujita M, Tsuruta R, Wakatsuki J, et al.: Methanol intoxication: differential diagnosis from anion gapincreased acidosis. Intern Med. 2004, 43:750-4.

11. Nazir S, Melnick S, Ansari S, Kanneh H: Mind the gap: a case of severe methanol intoxication . BMJ Case Rep. 2016, 2016:1-3.

12. Blanco M, Casado R, Vazquez F, Pumar JM: CT and MR imaging findings in methanol intoxication . AJNR Am J Neuroradiol. 2006, 27:452-4. 